\title{
Elastic Structure Preserving Impedance (ESPi) Control for Compliantly Actuated Robots
}

\author{
Manuel Keppler ${ }^{1}$, Dominic Lakatos ${ }^{1}$, Christian $\mathrm{Ott}^{1}$ and Alin Albu-Schäffer ${ }^{1,2}$
}

\begin{abstract}
We present a new approach for Cartesian impedance control of compliantly actuated robots with possibly nonlinear spring characteristics. It reveals a remarkable stiffness and damping range in the experimental evaluation. The most interesting contribution, is the way the desired closedloop dynamics is designed. Our control concept allows to add a desired stiffness and damping directly on the end-effector, while leaving the system structure intact. The intrinsic inertial and elastic properties of the system are preserved. This is achieved by introducing new motor coordinates that reflect the desired spring and damper terms. Theoretically, by means of additional motor inertia shaping it is possible to make the end-effector interaction behavior with respect to external loads approach, arbitrarily close, the interaction behavior that is achievable by classical Cartesian impedance control on rigid robots.

The physically motivated design approach allows for an intuitive understanding of the resulting closed-loop dynamics. We perform a passivity and stability analysis on the basis of a physically motivated storage and Lyapunov function.
\end{abstract}

\section{INTRODUCTION}

In the past, it turned out that the impact of joint elasticities on the controller design is of particular relevance for light weight robots as well as for industrial robots when precise and quick motions are required. In this case, it is common to rely on the reduced model [1] of a flexible joint robot that consists of the rigid body dynamics in feedback interconnection with the elastic actuator dynamics. Common causes of joint elasticities are gear flexibility or the compliance of a torque-force sensor. In these cases, the joint stiffness is quiet high and the robot dynamics is primarily dominated by the rigid body part. Consequently, several control approaches for such elastic joint robots started with a control law for the rigid body dynamics and used it as a desired torque to be controlled with the elastic actuator dynamics [2], [3], [4]. On the contrary, feedback linearization based approaches [5] designed the controller directly for the full flexible model without first designing a rigid body controller in an intermediate step. In both cases, a stable closed-loop systems is achieved, but its structure deviates significantly from the open loop system.

Starting with [8], [9] we aimed at developing passivitybased control approaches that preserve the intrinsic compliant dynamics of flexible joint robots. On robots with rather stiff joints these approaches show good performances. However, in highly compliant robots, it turned out that the vibration damping performances of these approaches, which rely on motor side damping and joint torque feedback, are

\footnotetext{
${ }^{1}$ The authors are with the Institute of Robotics and Mechatronics, German Aerospace Center (DLR), 82234 Oberpfaffenhofen, Germany.

e-mail: manuel.keppler@dlr.de

web: http://www.dlr.de/rmc/rm/en/staff/manuel.keppler

${ }^{2}$ Alin Albu-Schäffer is also with the Technical University Munich, Chair of Sensor Based Robots and Intelligent Assistance Systems, Department of Informatics, D-85748 Garching, Germany.
}

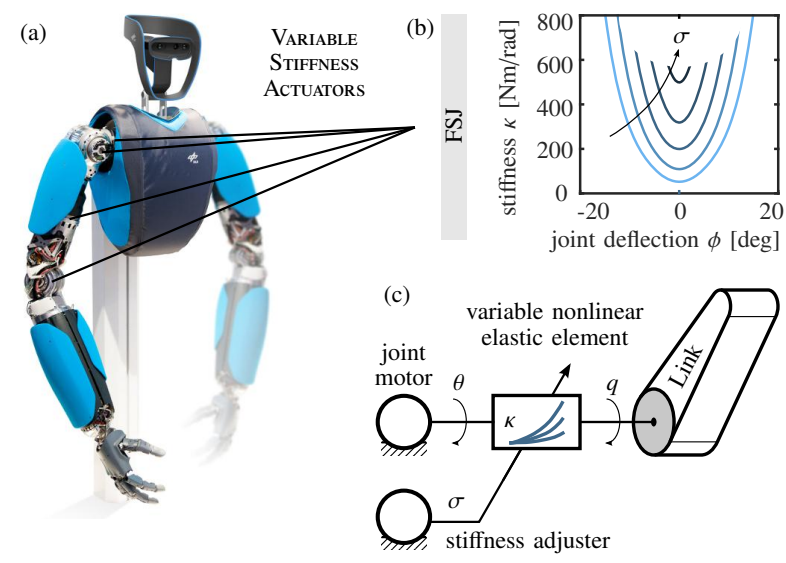

Fig. 1. Fig. 1a shows the DLR Hand Arm System [6]. The first four arm joints, namely the elbow and the three shoulder joints, are implemented by Floating Spring Joints (FSJ) [7]. A simplified working scheme is shown in Fig. 1c. The joint stiffness for various stiffness adjuster positions $\sigma=[0,2.5,5.0,7.5,10]$ deg is shown in Fig. $1 \mathrm{~b}$.

non-satisfactory. In robots featuring serial elastic actuators (SEA) or variable impedance actuators (VIA) one deliberately integrates a highly elastic element in the drive train. These type of robots gained popularity in the recent past as they offer a number of advantages such as improved impact tolerance [7], lower reflected inertia, more accurate and stable force control [10], energy storage that allows for explosive motions [11]. On the other hand, the highly elastic elements introduce intrinsic oscillatory dynamics into the plant. For this kind of highly elastic robots, we proposed a new passivity-based control approach [12], [13] that is based on the idea of preserving the elastic structure of the plant dynamics. In contrast to [9], it implements damping directly on the link side and not indirectly via motor damping on the motor side. From a conceptional point of view, these works are most closely related to the work [14] which proposes a controller that cancels the effects of gravity while retaining the dynamics structure. In [15] we adopted the design idea of our previous controllers to achieve Cartesian impedance control for robots with visco-elastic actuators. Despite their similar structure, visco-elastic actuators and SEA have crucially different control properties. Considering the link coordinates as output, visco-elastic systems have a relative degree of at most 3 . This leads to the situation that [15], in its most general from, requires dynamic state feedback. SEA systems, on the other hand, have a relative degree of 4 . In this paper we extend the concept of link-side damping of our previous works to full impedance control. In contrast to [15], static state feedback is sufficient. Impedance control was initially introduced by [16] and has been successfully applied on many robots in practice. It allows for stable interactions of the robot with its environment in a 
b) Achieved Closed-Loop Dynamics

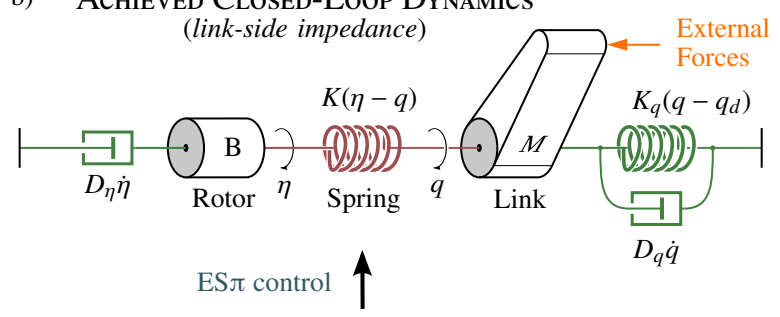

a)

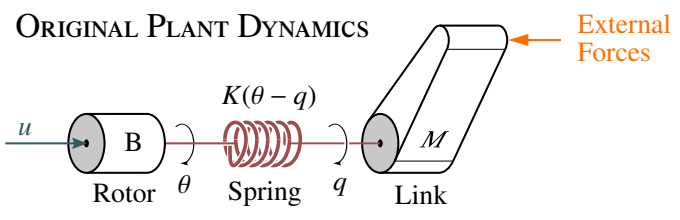

ESP control

c) Achieved Closed-Loop Dynamics

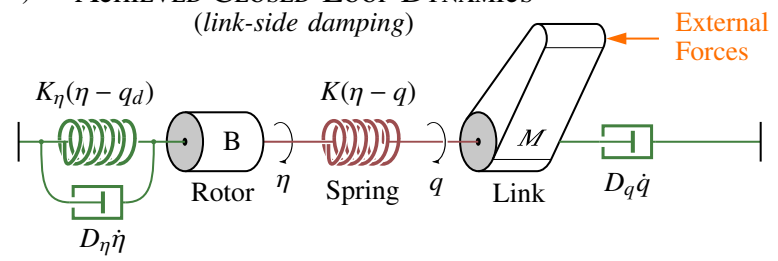

Fig. 2. (a) Graphical representation of a single linear-elastic joint as depicted in. (b) ES $\pi$ control achieves a full Impedance behavior directly on the link. (c) ESP control achieves damping directly on the link.

defined manner. Enabling Cartesian impedance control on highly elastic robots would increase their field of application significantly in our opinion. To the best of knowledge, this is the first Cartesian impedance controller that has been experimentally validated on multi-joint robot with highly elastic variable stiffness joints featuring highly nonlinear stiffness characteristics, see Fig. 1.

\section{The Basic Design IdeA}

This section presents the basic idea that underlies the proposed control concept. In order to keep the presentation simple, we consider a single joint with flexible transmission as shown in Fig. 2(a). The actuator with inertia $B$ is coupled via a linear-elastic element, with stiffness $K$, to the link inertia $M$. The motor and link coordinates are represented by $\theta$ and $q$, respectively. The displacement of the elastic element is described by the difference $\theta-q$. The control input $u$, is a generalized force acting on the motor inertia. The corresponding dynamics can be expressed as

$$
\begin{aligned}
M \ddot{q} & =K(\theta-q) \\
B \ddot{\theta}+K(\theta-q) & =u .
\end{aligned}
$$

The goal of impedance control is to achieve a desired dynamical behavior of the link with respect to an external generalized force.

We start by deriving a control law that adds a spring and damper term on the link side, while preserving the structure of the plant dynamics. This is achieved by introducing a new motor coordinate $\eta$ that satisfies

$$
K(\theta-q)=K(\eta-q) \underbrace{-D_{q} \dot{q}-K_{q}\left(q-q_{d}\right)}_{=: n}
$$

and reflects the desired link-side impedance behavior characterized by the spring stiffness $K_{q}$ and the viscous damping coefficient $D_{q} \cdot{ }^{1}$ The desired equilibrium point of the link is $q_{d}$. We choose the control law

$$
u=\bar{u}+B K^{-1} \ddot{n}+n,
$$

which leads, under consideration of (3), to the following intermediate dynamics

$$
\begin{aligned}
M \ddot{q} & =K(\eta-q)-D_{q} \dot{q}-K_{q}\left(q-q_{d}\right) \\
B \ddot{\eta}+K(\eta-q) & =\bar{u} .
\end{aligned}
$$

Next, we choose

$$
\bar{u}=-D_{\eta} \dot{\eta},
$$

such that the resulting closed-loop dynamics

$$
\begin{aligned}
M \ddot{q} & =K(\eta-q)-D_{q} \dot{q}-K_{q}\left(q-q_{d}\right) \\
B \ddot{\eta}+K(\eta-q) & =D_{\eta} \dot{\eta} .
\end{aligned}
$$

has the unique equilibrium point $q=\eta=q_{d}, \dot{q}=\dot{\eta}=0 .^{2}$ The corresponding graphical representation of the closed-loop dynamics is presented in Fig. 2(b). Clearly, we achieved or goal of modifying the link interaction behavior by adding a visco-elastic element directly on the link; compare Fig. 2(a) with Fig. 2(b).

In impedance control theory, a desired dynamical behavior usually is given by a differential equation of second order representing a mass-spring-damper system with mass $M$, desired stiffness $K_{q}$, and desired damping $D_{q}$. In our case, we achieved a double spring-mass-damper system with an extra mass $B$, and stiffness $K$, and damper $D_{\eta}$. For rather gentle interactions the link behavior is dominated by the spring $K_{q}$ and the damper $D_{q}$. Also the static equilibrium is solely defined by the spring $K_{q}$. Clearly, for forceful interactions the dynamics of the link deviates from the dynamics of the second order system. In this situation the resulting link behavior will be influenced also by the joint stiffness $K$, the actuator inertia $B$ and the damping factor $D_{\eta}$. Intuitively speaking, the deviation of the actual link behavior from the desired link behavior decreases with decreasing motor inertia $B$. By adding extra feedback of the virtual joint torque $K(\eta-q)$ one can shape the apparent motor inertia $B$ [9]. Naturally, the lower the shaped rotor inertia the better the approximation of the desired behavior. This concept will be put into concrete terms in Sec. III.

From the conceptual point of view, the ES $\pi$ control appears to be closely related to our previously proposed elastic structure preserving (ESP) control [13] as, loosely speaking, the visco-elastic elements are simply mirrored from the virtual motor side to the link side, cf. Fig. 2(b) and Fig. 2(c). But in practice, this difference has critical implications.

\footnotetext{
${ }^{1}$ For simplicity, we assume $K_{q}$ and $D_{q}$ to be constant.

${ }^{2}$ Note, according to (3), the corresponding equilibrium point in the original motor coordinates is $\left[q=q_{d}, \theta=q_{d}, \dot{q}=0, \dot{\theta}=0\right]$. Asymptotic stability of the equilibrium state of (4)-(5) can easily be shown by considering the Lyapunov function $V=1 / 2\left(M \dot{q}^{2}+B \dot{\eta}^{2}+K(\theta-q)^{2}+K_{q}\left(q-q_{d}\right)^{2}\right)$ and invoking La'Salles Theorem [17].
} 
Under ESP control, the link interaction behavior is primarily dominated by the intrinsic joint stiffness $K$ (usually $K_{\eta}>>$ $K$ ) and the damping coefficient $D_{q}$. Since $K$ is system inherent and cannot be changed (SEA) or just within small margins (VSA), the link interaction behavior is mostly fixed.

\section{Controller Design}

In this section we extend the basic idea presented in Sec. III-B to general robotic systems that feature nonlinear elastic transmissions, multiple joints and that are subject to gravity. First, we treat the case of Cartesian impedance control. Joint-level impedance control is a special case thereof and can be easily derived from it. Before we start, we set the scene by introducing the class of robots we consider.

\section{A. Robot Dynamics}

Throughout this paper, we consider a simplified model of a $n$-link robot with compliant joints, which is based on the model proposed by Spong in [1]. It is given by

$$
\begin{aligned}
\boldsymbol{M}(\boldsymbol{q}) \ddot{\boldsymbol{q}}+\boldsymbol{C}(\boldsymbol{q}, \dot{\boldsymbol{q}}) \dot{\boldsymbol{q}} & =\boldsymbol{\psi}(\boldsymbol{\theta}-\boldsymbol{q})-\boldsymbol{g}(\boldsymbol{q})+\tau_{e x t} \\
\boldsymbol{B} \ddot{\boldsymbol{\theta}}+\boldsymbol{\psi}(\boldsymbol{\theta}-\boldsymbol{q}) & =\boldsymbol{u} .
\end{aligned}
$$

Herein, $\boldsymbol{q} \in \mathbb{R}^{n}$ and $\boldsymbol{\theta} \in \mathbb{R}^{n}$ represent the link angles and motor angles, respectively. The symmetric and positive definite inertia matrix of the rigid links is denoted by $M \in$ $\mathbb{R}^{n \times n}$. The constant, diagonal and positive definite matrix $\boldsymbol{B} \in \mathbb{R}^{n \times n}$ consists of the actuator inertias. ${ }^{3}$ These conditions are fulfilled for all pure rotational and pure prismatic joint robots and in some special cases for robots that feature a mix of rotational and prismatic joints, see [18] for an in-depth discussion. The gravitational forces are represented by $\boldsymbol{g}(\boldsymbol{q})$, and $\boldsymbol{C}(\boldsymbol{q}, \dot{\boldsymbol{q}}) \dot{\boldsymbol{q}}$ denotes the Coriolis and centrifugal forces. As suggested by [19], we define the $n \times n$ matrix $\boldsymbol{C}(\boldsymbol{q}, \dot{\boldsymbol{q}})$ via the Christoffel symbols, such that the matrix $\dot{M}(\boldsymbol{q})-2 \boldsymbol{C}(\boldsymbol{q}, \dot{\boldsymbol{q}})$ is skew symmetric for all $(\boldsymbol{q}, \dot{\boldsymbol{q}}) \in \mathbb{R}^{n} \times \mathbb{R}^{n}$ [20]. Only the motor coordinates $\boldsymbol{\theta}$ can be directly actuated via the generalized motor forces $\boldsymbol{u} \in \mathbb{R}^{n}$, which serve as the control input. The link coordinates $\boldsymbol{q}$ can only be indirectly actuated via the elastic torques $\psi(\phi)$, which are, in general, nonlinear functions of the spring deflection $\phi=\boldsymbol{\theta}-\boldsymbol{q}$. This form of under-actuation represents the major challenge in the control of the states $\boldsymbol{q}$ and $\dot{\boldsymbol{q}}$. Assumptions on the spring potential $U_{s}$ and assumptions specific for the Cartesian impedance control case are given in the Appendix X.

\section{B. Generalization of the Design Idea}

In the Idea Sec. II we added spring and damper terms to the link. Here, we aim to extend the link dynamics with terms that are due to Cartesian springs and dampers. This would allow the user to impose a desired dynamical behavior between the robot end-effector motion and external generalized forces $\tau_{e x t}$. This relationship will be described in terms of the end-effector coordinates $\boldsymbol{x}$. We consider the Cartesian spring and damper forces to be of the form

$$
\boldsymbol{F}_{x}(\tilde{\boldsymbol{x}}, \dot{\boldsymbol{x}})=-\left(\boldsymbol{D}_{x} \dot{\boldsymbol{x}}+\frac{\partial U_{x}(\tilde{\boldsymbol{x}})}{\partial \boldsymbol{x}}\right),
$$

where $\boldsymbol{D}_{x}$ specifies the desired damping and $\frac{\partial U_{s}(\tilde{\boldsymbol{x}})}{\partial \boldsymbol{x}}$ the spring forces that are derived from a potential function $U_{s}$. The

\footnotetext{
${ }^{3}$ When talking about motor inertias, we refer to the sum of all the motorside inertias reflected through the gearboxes to the compliant element side.
}

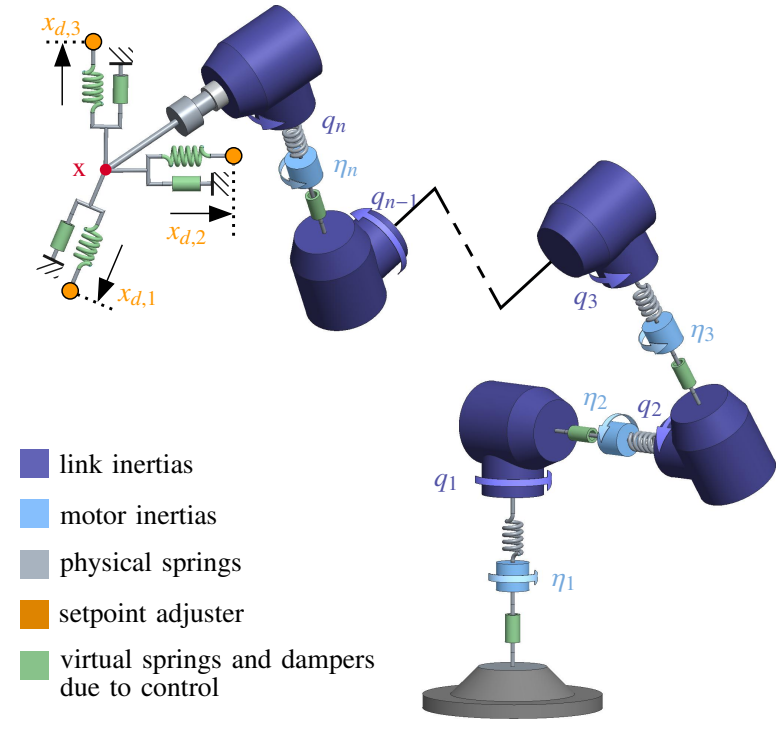

Fig. 3. Achieved closed-loop dynamics via ES $\pi$ control for a n-joint flexible joint robot. We directly added a Cartesian spring (specified by $\left.\frac{\partial U_{x}(\tilde{\boldsymbol{x}})}{\partial \boldsymbol{x}}\right)$ and damper $\boldsymbol{D}_{x}$ on the end-effector in order to modify the Impedance.

position errors in operational space $\tilde{\boldsymbol{x}}$ are denoted by $\tilde{\boldsymbol{x}}:=$ $\boldsymbol{x}-\boldsymbol{x}_{d}$. The following assumptions have to be met.

Assumption 1. Let $\beta$ be a strictly positive constant. The potential function $U_{x}: \mathbb{R}^{n} \rightarrow \mathbb{R}$ has to be lower bounded as follows ${ }^{4}$

$$
U_{x}(\tilde{\boldsymbol{x}})-U_{x}(\mathbf{0}) \geq \beta\|\tilde{\boldsymbol{x}}\|^{2} .
$$

Assumption 2. The damping matrix $\boldsymbol{D} \in \mathbb{R}^{n \times n}$ is symmetric, positive definite and bounded. ${ }^{5}$

The operational space forces $\boldsymbol{F}_{x}$ can be transformed to the corresponding joint $\boldsymbol{\tau}_{x}$ forces according to [21] via

$$
\boldsymbol{\tau}_{x}(\tilde{\boldsymbol{x}}, \dot{\boldsymbol{x}})=\boldsymbol{J}^{\mathrm{T}}(\boldsymbol{q}) \boldsymbol{F}_{x} .
$$

The Jacobian is introduced in the Appendix X.In analog fashion, we can relate external forces $\boldsymbol{F}_{\text {ext }}$ to external torques $\tau_{e x t}$ via $\boldsymbol{\tau}_{\text {ext }}=\boldsymbol{J}^{\mathrm{T}}(\boldsymbol{q}) \boldsymbol{F}_{\text {ext }}$. We now aim to derive a controller $\boldsymbol{u}$ in (7) such that the behavior of the resulting compensated system is equivalent to the following desired dynamics

$$
\begin{aligned}
\boldsymbol{M}(\boldsymbol{q}) \ddot{\boldsymbol{q}}+\boldsymbol{C}(\boldsymbol{q}, \dot{\boldsymbol{q}}) \dot{\boldsymbol{q}} & =\boldsymbol{\psi}(\boldsymbol{\eta}-\boldsymbol{q})+\tau_{x}(\tilde{\boldsymbol{x}}, \dot{\boldsymbol{x}})+\tau_{\text {ext }} \\
\boldsymbol{B} \ddot{\boldsymbol{\eta}}+\boldsymbol{\psi}(\boldsymbol{\eta}-\boldsymbol{q}) & =-\boldsymbol{D}_{\eta} \dot{\boldsymbol{\eta}} .
\end{aligned}
$$

Note, we extended the link-side dynamics solely with the impedance terms $\tau_{x}$ and canceled gravity. The inertial and elastic properties of the plant are preserved. The new motor coordinates $\boldsymbol{\eta} \in \mathbb{R}^{n}$ reflect this desired link behavior. ${ }^{6}$

\footnotetext{
${ }^{4}$ One popular potential function that satisfies this assumption is $\frac{1}{2} \tilde{\boldsymbol{x}}^{\mathrm{T}} \boldsymbol{K}_{x} \tilde{\boldsymbol{x}}$, with $\boldsymbol{K}_{x} \in \mathbb{R}^{n \times n}$ being a constant, positive definite matrix.

${ }^{5}$ The user can even choose $\boldsymbol{D}$ to be state and explitly state-dependent as long as the conditions of the implicit function theorem are satisfied such that $\boldsymbol{\eta}$ in (13) can be expressed in terms of the other variables.

${ }^{6}$ The desired dynamics (11)-(12) is written mixed in link coordinates $\boldsymbol{q}$ and operational space coordinates $\boldsymbol{x}$, even though our desired interaction behavior is defined in operational space state variables $\tilde{\boldsymbol{x}}, \dot{\boldsymbol{x}}$ via (8). We maintain this mixed notation throughout the paper since many terms can be written clearer and simpler as function of $\boldsymbol{q}$. In the end, $\boldsymbol{q}$ and $\dot{\boldsymbol{q}}$ can always be thought of being substituted by $q=G_{1}(x)$ and $\dot{q}=J_{1}\left(G_{1}(x)\right) \dot{x}$.
} 


\section{Achieving the Desired Dynamics}

We start by imposing equivalence of (6) and (11) to find an implicit relation

$$
\boldsymbol{\psi}(\boldsymbol{\theta}-\boldsymbol{q})=\psi(\boldsymbol{\eta}-\boldsymbol{q})+\underbrace{\boldsymbol{g}(\boldsymbol{q})+\tau_{x}(\tilde{\boldsymbol{x}}, \dot{\boldsymbol{x}})}_{=: \boldsymbol{n}\left(\boldsymbol{x}_{d}, \boldsymbol{x}, \dot{\boldsymbol{x}}\right)}
$$

between the new motor coordinates $\boldsymbol{\eta}$ and the original motor coordinates $\boldsymbol{\theta} .^{7}$ Next, we want to achieve equivalence of (7) and (12). To this end, we transform the motor dynamics (7) into the new coordinates $\boldsymbol{\eta}$ which requires the second time derivative of $\boldsymbol{\theta}$ as function of $\boldsymbol{\eta}$. We differentiate the coordinate transformation with respect to time and obtain after rearranging some terms

$$
\begin{aligned}
\dot{\theta} & =A\left(x_{d}, x, \dot{x}, \theta\right) \dot{\eta}+\boldsymbol{a} \\
\boldsymbol{A}\left(\boldsymbol{x}_{d}, \boldsymbol{x}, \dot{\boldsymbol{x}}, \boldsymbol{\theta}\right) & :=\kappa 1(\theta-\boldsymbol{\theta}) \boldsymbol{\kappa}(\boldsymbol{\eta}-\boldsymbol{q}) \\
\boldsymbol{a}\left(\boldsymbol{q}_{d}, \boldsymbol{q}, \dot{\boldsymbol{q}}, \ddot{\boldsymbol{q}}, \boldsymbol{\theta}\right) & :=\left(\boldsymbol{I}_{n \times n}-\boldsymbol{A}(\boldsymbol{q}, \dot{\boldsymbol{q}}, \boldsymbol{\theta})\right) \dot{\boldsymbol{q}}+\kappa 1(\theta-\boldsymbol{q}) \dot{\boldsymbol{n}}(\boldsymbol{q}, \dot{\boldsymbol{q}}, \boldsymbol{\theta})
\end{aligned}
$$

Herein, $\boldsymbol{A}$ can be interpreted as a set-point ${ }^{8}$ and statedependent Jacobian matrix that constitutes a ratio between the local stiffnesses of the nonlinear springs of the original and the reference system. By deriving (14) with respect to time, we get a relation between the accelerations of the old and new motor coordinates

$$
\ddot{\theta}=A \ddot{\eta}+\dot{A} \dot{\eta}+\dot{a} .
$$

The relations (13) and (15) allow us to perform a coordinate transformation $[\boldsymbol{\theta}, \boldsymbol{q}] \mapsto[\boldsymbol{\eta}, \boldsymbol{q}]$ for the robot dynamics (6)-(7). Obviously, for the link dynamics (6) we obtain the desired dynamics (11). For the transformed motor dynamics we get

$$
B A \ddot{\eta}+B \dot{A} \dot{\eta}+B \dot{a}+\psi(\eta-q)+n=u
$$

We now choose the control input

$$
\begin{aligned}
\boldsymbol{u}= & \boldsymbol{B}(\dot{\boldsymbol{A}} \dot{\boldsymbol{\eta}}+\dot{\boldsymbol{a}})+\boldsymbol{n}-\boldsymbol{B} \boldsymbol{A} \boldsymbol{B}_{1} K_{\eta} \dot{\boldsymbol{\eta}} \\
& +\left(\boldsymbol{I}_{n \times n}-\boldsymbol{B} \boldsymbol{A} \boldsymbol{B}_{1}\right) \psi(\boldsymbol{\eta}-\boldsymbol{q}),
\end{aligned}
$$

in order to obtain the desired motor dynamics (12).

Remark 1. Control law (16) is significantly simpler than a corresponding feedback linearization based control law, which would contain the first and second time derivatives of both the inertia matrix $\boldsymbol{M}(\boldsymbol{q})$ and the Coriolis and centrifugal forces $C(\boldsymbol{q}, \dot{\boldsymbol{q}}) \dot{\boldsymbol{q}}$.

Remark 2. In theory, we can realize arbitrary torques $\boldsymbol{n}$, cf. (13), on the links. With the only assumption, that their time derivatives up to the second order are known. This might lead to interesting applications of the $\mathrm{ES} \pi$ control concept in future such as implementation of reactive collision avoidance methods, virtual force fields and virtual walls.

\footnotetext{
${ }^{7}$ For the case that the inverse function of $\boldsymbol{\psi}$ is not analytically available, one has to solve (13) for $\boldsymbol{\eta}$ numerically. The existence of such a solution is ensured, under assumptions made above, by the implicit function theorem.

${ }^{8}$ The set-point dependence is due to the coordinate transformation that is parameterized by $\boldsymbol{q}_{d}$.
}

\section{Motor Inertia Shaping}

In Section II we elaborated on the implications of motor inertia shaping on the basis of a single joint robot. The implications can be directly transferred to the multi joint case. By changing the control law from (16) slightly to

$$
\begin{aligned}
\boldsymbol{u}^{*}= & \boldsymbol{B}(\dot{\boldsymbol{A}} \dot{\boldsymbol{\eta}}+\ddot{\boldsymbol{a}})+\boldsymbol{n}-\boldsymbol{B} \boldsymbol{A} \check{\boldsymbol{B}}_{1} K_{\eta} \dot{\boldsymbol{\eta}} \\
& +\left(\boldsymbol{I}_{n \times n}-\boldsymbol{B} \boldsymbol{A} \check{\boldsymbol{B}}_{1}\right) \psi(\eta-q),
\end{aligned}
$$

we obtain the following closed-loop motor dynamics

$$
\check{B} \ddot{\eta}+\psi(\eta-q)=-D_{\eta} \dot{\eta}
$$

where we scaled the motor inertia from $\boldsymbol{B}$ to $\check{\boldsymbol{B}}$

Remark 3. Let us assume that $\boldsymbol{B}$ and $\check{\boldsymbol{B}}$ are related via a scalar factor $\beta>0$ such that $\check{\boldsymbol{B}}=\beta \boldsymbol{B}$, and that $\boldsymbol{A}$ is diagonal. In that case, we have $\boldsymbol{B} \boldsymbol{A} \check{\boldsymbol{B}}_{1}=\beta_{1} \boldsymbol{A}$ and motor inertia shaping can be thought of as a linear scaling of the feedback of the virtual spring torques $\boldsymbol{\psi}(\boldsymbol{\eta}-\boldsymbol{q})$ and the motor damping torques $K_{\eta} \dot{\boldsymbol{\eta}}$.

\section{Closed-Loop Dynamics in Cartesian Coordinates}

For the passivity analysis (Sec. V) and the stability analysis in (Sec. VI) of the closed-loop dynamics (11) and (18), it is advantageous to transform the dynamics equations into task coordinates. By pre-multiplying (11) with $\boldsymbol{J}^{-\mathrm{T}}(\boldsymbol{q})$ and substituting $\ddot{\boldsymbol{q}}$ and $\dot{\boldsymbol{q}}$, which can be obtained from the first and second the time derivatives of (27), we get for the closedloop link dynamics

$$
\boldsymbol{\Lambda}_{x}(\boldsymbol{x}) \ddot{\boldsymbol{x}}+\boldsymbol{\mu}_{x}(\boldsymbol{x}, \dot{\boldsymbol{x}}) \dot{\boldsymbol{x}}=\boldsymbol{J}^{-\mathrm{T}} \boldsymbol{\psi}(\boldsymbol{\eta}-\boldsymbol{q})+\boldsymbol{F}_{x}(\tilde{\boldsymbol{x}}, \dot{\boldsymbol{x}})+\boldsymbol{F}_{\text {ext }}
$$

where

$$
\begin{aligned}
\Lambda_{x}(x) & :=J^{-\mathrm{T}}(q) M(q) J_{1}(q) \\
\mu_{x}(x, \dot{x}) & :=J^{-\mathrm{T}}(q)\left(C(q, \dot{q})-M(q) J_{1}(q) \dot{J}(q)\right) J_{1}(q)
\end{aligned}
$$

are the link-side inertia and Coriolis/centrifugal matrices expressed in $\boldsymbol{x}$ coordinate. The inertia matrix $\boldsymbol{\Lambda}$ is symmetric and positive definite. Analog to the joint case, the matrix $\boldsymbol{\Lambda}_{x}(\boldsymbol{x})-2 \boldsymbol{\mu}_{x}(\boldsymbol{x}, \dot{\boldsymbol{x}})$ is skew-symmetric for all $\boldsymbol{x} \in$ and all $\dot{\boldsymbol{x}} \in \mathbb{R}^{m}$

Remark 4. By considering $G$, see Appendix $\mathrm{X}$, as the identity mapping, control law (16) reduces to a joint-level impedance controller. The corresponding closed-loop dynamics can therefore be considered as a special case of (18)-(19). As a direct consequence, all passivity and stability statements in Sec. V and Sec. VI directly apply to the joint impedance case.

\section{Passivity Analysis}

In this section, we analyze the passivity properties of the closed-loop systems (18)-(19). The physically motivated design approach of the reference dynamics, c.f. Fig. 2(b), suggest the following intuitive storage functions

$$
\begin{aligned}
S_{x}(\tilde{\boldsymbol{x}}, \dot{\boldsymbol{x}}) & =\frac{1}{2} \dot{\boldsymbol{x}}^{\mathrm{T}} \boldsymbol{\Lambda}_{x}(\boldsymbol{q}) \dot{\boldsymbol{x}}+U_{x}(\tilde{\boldsymbol{x}}), \\
S_{\eta}(\boldsymbol{x}, \boldsymbol{\eta}, \dot{\boldsymbol{\eta}}) & =\frac{1}{2} \dot{\boldsymbol{\eta}}^{\mathrm{T}} \boldsymbol{B} \dot{\boldsymbol{\eta}}+U_{s}(\boldsymbol{\eta}-\boldsymbol{q}) .
\end{aligned}
$$

The sum $S=S_{x}+S_{\eta}$ comprises the total virtual kinetic and potential energy of the closed-loop system (18)-(19). The 


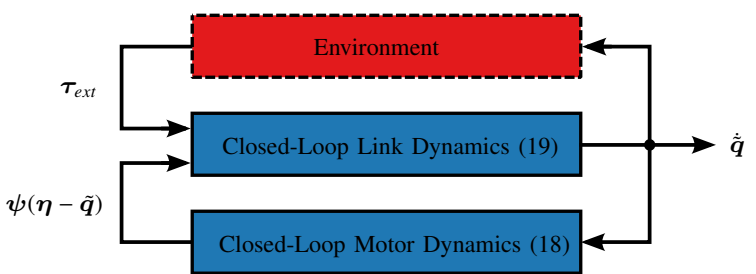

Fig. 4. The closed-loop dynamics (18)-(19) can be interpreted as an interconnection of passive subsystems.

time derivatives of the storage function $S_{x}$ and $S_{\eta}$, expressed along the solutions of the closed-loop dynamics, give

$$
\begin{aligned}
\dot{S}_{x}(\tilde{\boldsymbol{x}}, \dot{\boldsymbol{x}}) & =-\dot{\boldsymbol{x}}^{\mathrm{T}} \boldsymbol{D} \dot{\boldsymbol{x}}+\dot{\boldsymbol{x}}^{\mathrm{T}} \boldsymbol{J}^{-\mathrm{T}}(\boldsymbol{q}) \boldsymbol{\psi}(\boldsymbol{\eta}-\boldsymbol{q})+\dot{\boldsymbol{x}}^{\mathrm{T}} \boldsymbol{J}^{-\mathrm{T}}(\boldsymbol{q}) \tau_{e x t}, \\
\dot{S}_{\eta}(\boldsymbol{x}, \boldsymbol{\eta}, \dot{\boldsymbol{\eta}}) & =-\dot{\boldsymbol{\eta}}^{\mathrm{T}} \boldsymbol{K}_{d} \dot{\boldsymbol{\eta}}-\dot{\boldsymbol{q}}^{\mathrm{T}} \boldsymbol{\psi}(\boldsymbol{\eta}-\boldsymbol{q}) .
\end{aligned}
$$

We can identify three kind of terms. First, each of the quadratic forms represents the power dissipation that is due to a virtual damper. Second, the term $\dot{\boldsymbol{q}}^{\mathrm{T}} \boldsymbol{\psi}(\boldsymbol{q}-\boldsymbol{\eta})$ correspond to interconnection ports between the motor and link dynamics. ${ }^{9}$ Third, $\dot{\boldsymbol{x}}^{\mathrm{T}} \boldsymbol{F}_{\text {ext }}$ represents an interconnection port that allows energy exchange between the robot and its environment, see Fig. 4. The result so far motivate the following proposition:

Proposition 1. The closed-loop system (18)-(19) represents a passive map from external forces $\boldsymbol{F}_{\text {ext }}$ to the velocities $\dot{\boldsymbol{x}}$.

Proof. The time derivatives of the storage functions $S$ is given by

$$
\dot{S}(\boldsymbol{x}, \dot{\boldsymbol{x}}, \boldsymbol{\eta}, \dot{\boldsymbol{\eta}})=-\dot{\boldsymbol{x}}^{\mathrm{T}} \boldsymbol{D}_{x} \dot{\boldsymbol{x}}-\dot{\boldsymbol{\eta}}^{\mathrm{T}} \boldsymbol{K}_{d} \dot{\boldsymbol{\eta}}+\dot{\boldsymbol{q}}^{\mathrm{T}} \boldsymbol{\tau}_{\text {ext }} \leq \dot{\boldsymbol{x}}^{\mathrm{T}} \boldsymbol{F}_{\text {ext }}
$$

\section{Stability Analysis}

Here we present a formal stability analysis of the closedloop system (18)-(19) in absence of external generalized forces $\boldsymbol{F}_{x}$. We consider $\boldsymbol{z}=\left[\begin{array}{llll}\boldsymbol{x}^{\mathrm{T}} & \boldsymbol{\eta}^{\mathrm{T}} & \dot{\boldsymbol{x}}^{\mathrm{T}} & \dot{\boldsymbol{\eta}}^{\mathrm{T}}\end{array}\right]^{\mathrm{T}}$ as the system state. By virtue of Assumption 3, follows that

$$
\boldsymbol{z}_{0}=\left[\begin{array}{llll}
x_{d}^{\mathrm{T}} & \boldsymbol{G} \mathbf{1}\left(\boldsymbol{x}_{d}\right)^{\mathrm{T}} & \mathbf{0}^{\mathrm{T}} & \mathbf{0}^{\mathrm{T}}
\end{array}\right]^{\mathrm{T}}
$$

is a unique equilibrium state of (18)-(19). The time derivative of the storage function $S$, cf. (22), motivates the following proposition.

Proposition 2. Consider the closed-loop dynamics (18)-(19) in absence of external forces $\boldsymbol{F}_{\text {ext }}$. The equilibrium point (23) is asymptotically stable. ${ }^{10}$

Proof. Consider the following Lyapunov function candidate $V(\tilde{\boldsymbol{x}}, \dot{\boldsymbol{x}}, \boldsymbol{\eta}, \dot{\boldsymbol{\eta}})=S(\tilde{\boldsymbol{x}}, \dot{\boldsymbol{x}}, \boldsymbol{\eta}, \dot{\boldsymbol{\eta}})$. Clearly, $V$ is a positive definite

\footnotetext{
${ }^{9}$ Recall, that $\dot{\boldsymbol{x}}^{\mathrm{T}} \boldsymbol{J}^{-\mathrm{T}}(\boldsymbol{q})=\dot{\boldsymbol{q}}^{\mathrm{T}}$.

${ }^{10}$ The stability analysis in the physical $\boldsymbol{x}$ and virtual $\boldsymbol{\eta}$ coordinates is sufficient. Once, asymptotic stability of the equilibrium point $\boldsymbol{z}_{0}$ has been shown, one can deduce via (13) the corresponding equilibrium point in the $\boldsymbol{x}$ and $\boldsymbol{\theta}$ coordinates. It turns out to be

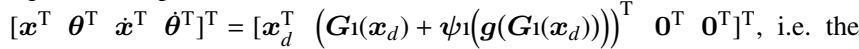
equilibrium position of $\boldsymbol{\theta}$ is shifted by $\psi_{1}\left(\boldsymbol{g}\left(G_{1}\left(x_{d}\right)\right)\right)$ relative to equilibrium position of $\boldsymbol{\eta}$, which is precisely the deflection of the intrinsic spring due to gravity as expected.
}

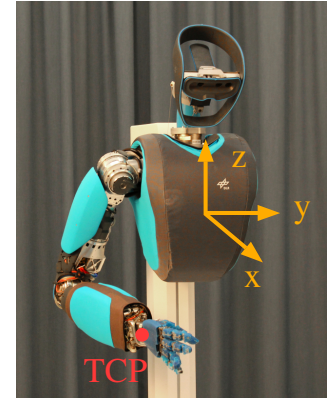

Fig. 5. Robot David in the initial configuration, i.e. $\boldsymbol{x} \approx \boldsymbol{x}_{d}$. All forces and Cartesian coordinates are expressed in the coordinate system shown.

function of the state $z$ due to Assumption 3. Its time derivative along the solutions of (18)-(19) is given by

$$
\dot{V}(\tilde{\boldsymbol{x}}, \dot{\boldsymbol{x}}, \boldsymbol{\eta}, \dot{\boldsymbol{\eta}})=-\dot{\boldsymbol{x}}^{\mathrm{T}} \boldsymbol{D}_{x} \dot{\boldsymbol{x}}-\dot{\boldsymbol{\eta}} \boldsymbol{K}_{d} \dot{\boldsymbol{\eta}} \leq 0,
$$

which is a negative semi-definite function. This allows us to invoke LaSalle's invariance principle to conclude asymptotic stability of the equilibrium point (23).

\section{EXPERIMENTS}

In this section, we present four experiments for the validation of the proposed control laws. All experiments have been conducted on the first four VSA joints of the DLR Hand Arm System [6], also known as David. The experimental setup is shown in Fig. 5.

It is important to point out that $\mathrm{ES} \pi$ control is not limited to this particular type of VSA as implemented in David. $\mathrm{ES} \pi$ control is applicable to any robot featuring VSA or serial elastic actuators (SEA) that fulfill Assumption 3, which is the case for a huge class of robots. These assumptions can be weakened by taking the results of [22] into account. Throughout all experiments the motor inertia is scaled down by a factor of $\beta=0.3$ which lowers the parasitic effect of the motor inertias. For the experiments where a human subject interacts with the robot, we estimate the forces that are exerted on the robot by an momentum based observer [23]. The estimated external forces $\boldsymbol{F}_{\text {ext }}$ and torques $\boldsymbol{\tau}_{\text {ext }}$ are used for validation purposes only and we filtered these signals offline with a Butterworth filter with a $-3 \mathrm{~dB}$ cutoff frequency at $10 \mathrm{~Hz}$. The damping matrices $\boldsymbol{K}_{x}, \boldsymbol{K}_{q}$ and $\boldsymbol{K}_{d}$ are designed in an analog way as described in [24]. In the following, $\boldsymbol{\xi}_{x}, \boldsymbol{\xi}_{q}$ and $\boldsymbol{\xi}_{\eta}$ represent the respective modal damping factors for $\boldsymbol{K}_{x}, \boldsymbol{K}_{q}$ and $\boldsymbol{K}_{d}$ (zero values mean no damping). A video that demonstrates the performance of $\mathrm{ES} \pi$ control on the DLR Hand Arm System is provided under the following link: https://youtu.be/sbhiNNIxMNQ.

\section{A. Interaction with a Human User}

The first experiment verifies the accuracy of the controlled Cartesian stiffness during the interaction with a human subject. The user grasps the robot at the end-effector and exerts forces - mainly in the $\mathrm{x}-\mathrm{y}$ plane - and therefore deflects the TCP from its equilibrium position. The initial robot configuration is shown in Fig. 5. The interaction behavior is determined by the commanded values of the diagonal stiffness matrix $\boldsymbol{K}_{x}$. Its values are summarized in the first row of Table I. The estimated forces that are exerted by the user are displayed in Fig. 6a. The corresponding deflection plots are contained in Fig. 6b. In order to validate the 
TABLE I

CONTROLLER PARAMETERS FOR EXPERIMENT 1 AND 3.

\begin{tabular}{|c|c|c|c|c|c|c|}
\hline $\boldsymbol{K}_{x, 11}$ & $\boldsymbol{K}_{x, 22}$ & $\boldsymbol{K}_{x, 33}$ & \multirow[t]{2}{*}{$\xi_{x}$} & \multirow[t]{2}{*}{$\xi_{\eta}$} & \multirow[t]{2}{*}{$\beta$} & \multirow{2}{*}{$\begin{array}{r}\sigma_{i=1 \ldots} \\
\operatorname{deg}\end{array}$} \\
\hline \multicolumn{3}{|c|}{ Cartesian stiffness in $\mathrm{N} \mathrm{m}^{-1}$} & & & & \\
\hline 0 & 60 & 3000 & 0 & 0 & 0.3 & 5 \\
\hline 1500 & 600 & 1500 & $0.6 / 0$ & 0.05 & 0.3 & 5 \\
\hline
\end{tabular}
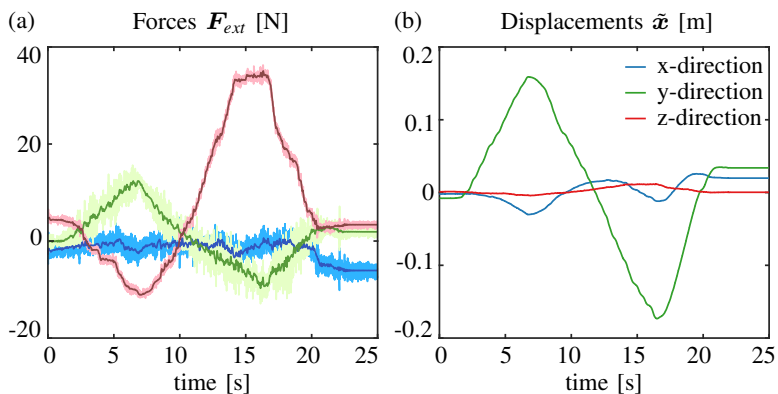

Fig. 6. Forces exerted by the user on the TCP of the robot (raw and filtered signals of forces) for the Cartesian impedance experiment.

achieved stiffness behavior, the applied forces in $e_{x}$ and $e_{y^{-}}$ direction are plotted versus the corresponding displacements in Fig. 7. The static characteristics that corresponds to the desired stiffness behavior are shown by the black dotted lines. The hysteresis-like deviation from the desired quasistatic behavior is in some degree due to uncompensated linkside friction and due to inaccuracies in the estimation of the external disturbances. The static error of the estimated forces, in absence of external forces, is in the magnitude of up to $8 \mathrm{~N}$, see e.g. Fig. $6 \mathrm{a}$ at $t=0$. The same experiment has been repeated for the joint-level ES $\pi$ controller with the settings given in the first row of Tab. II. The estimated external torques $\tau_{\text {ext }}$ applied by the user are shown in Fig. 8. The plot in Fig. 9 displays $\tau_{\text {ext }}$ over the corresponding link deflections $\tilde{\boldsymbol{q}}$. Figure 10 shows the local joint stiffness
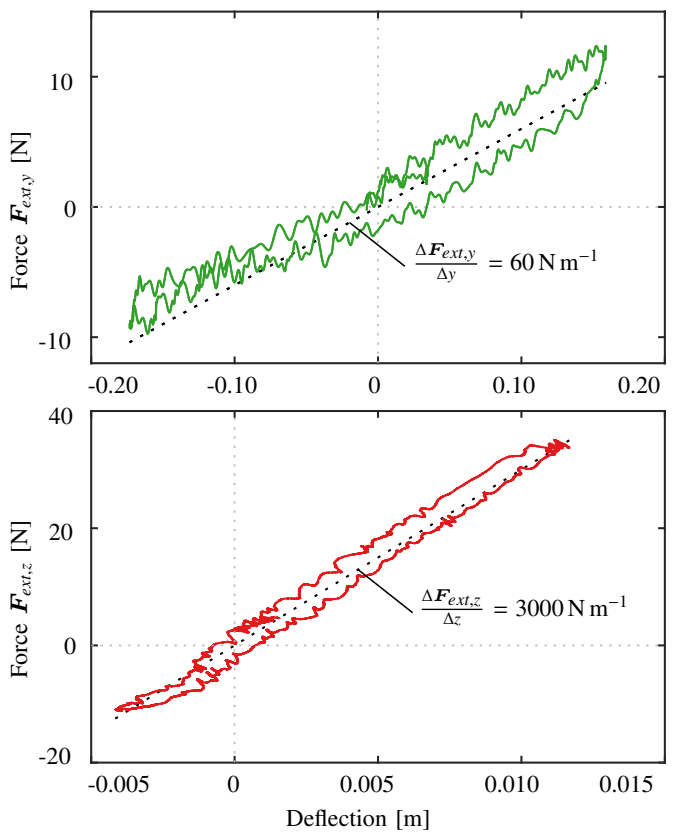

Fig. 7. Forces exerted in the $x-y$-plane versus the corresponding deflections in the $x-y$-plane. The black dashed lines correspond to each of the desired stiffness behaviors for the quasi-static case.
TABLE II

CONTROLLER PARAMETERS FOR EXPERIMENT 2 AND 4.

\begin{tabular}{|c|c|c|c|c|c|c|c|}
\hline $\boldsymbol{K}_{q, 11}$ & $\boldsymbol{K}_{q, 22}$ & $\boldsymbol{K}_{q, 33}$ & $\boldsymbol{K}_{q, 44}$ & \multirow[t]{2}{*}{$\xi_{q}$} & \multirow[t]{2}{*}{$\xi_{\eta}$} & \multirow[t]{2}{*}{$\beta$} & \multirow{2}{*}{$\begin{array}{r}\sigma_{i=1 \ldots 4} \\
\operatorname{deg}\end{array}$} \\
\hline \multicolumn{4}{|c|}{ Angular stiffness in $\mathrm{N} \mathrm{m} \mathrm{rad}^{-1}$} & & & & \\
\hline 1000 & 500 & 150 & 50 & 0.1 & 0.05 & 0.3 & 5 \\
\hline 800 & 800 & 800 & 800 & 0.6 & 0.05 & 0.3 & 5 \\
\hline
\end{tabular}

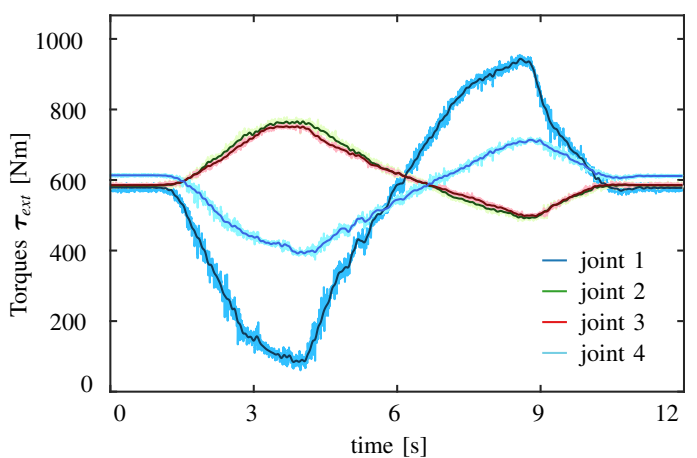

Fig. 8. External torques $\boldsymbol{\tau}_{\text {ext }}$ applied by the user (raw and filtered signals) for the joint-level impedance experiment.

values of the physical springs, i.e. $\boldsymbol{\kappa}(\boldsymbol{\theta}-\boldsymbol{q})$. For comparison, the commanded link-side stiffness values $\boldsymbol{K}_{q}$ are shown as well. For this particular experiment, the commanded - and achieved, cf. Fig. 9 - link-side stiffnesses are up to 5 times higher than the stiffnesses of the real physical stiffness.

\section{B. Disturbance Rejection Behavior}

The third experiment shows the disturbance rejection performance of the Cartesian ES $\pi$ controller for two different damping settings $\left(\xi_{x}=0.6\right.$ and $\left.\xi_{x}=0\right)$ and compares them to the well known motor PD controller by Tomei [25]. ${ }^{11}$ The settings are given in row two of Tab. I. In this experiment, the TCP of the robot was deflected by the user from its initial configuration and then quickly released. The results are shown in Fig. 11. The respective points in time when the TCP is released are indicated by orange triangles. For the well-damped case the disturbance of approx. $9 \mathrm{~cm}$ is rejected within $0.28 \mathrm{~s}$ with basically no overshooting. The graphs of the corresponding internal joint torques $\boldsymbol{\psi}(\boldsymbol{\theta}-\boldsymbol{q})$ show equally nice convergence behavior. This means, once

\footnotetext{
${ }^{11}$ The proportional gains were set to $8000 \mathrm{~N} \mathrm{~m} \mathrm{rad}^{-1}$ and the damping gains to $250 \mathrm{~N} \mathrm{rad}^{-1} \mathrm{~s}$ for each joint
}

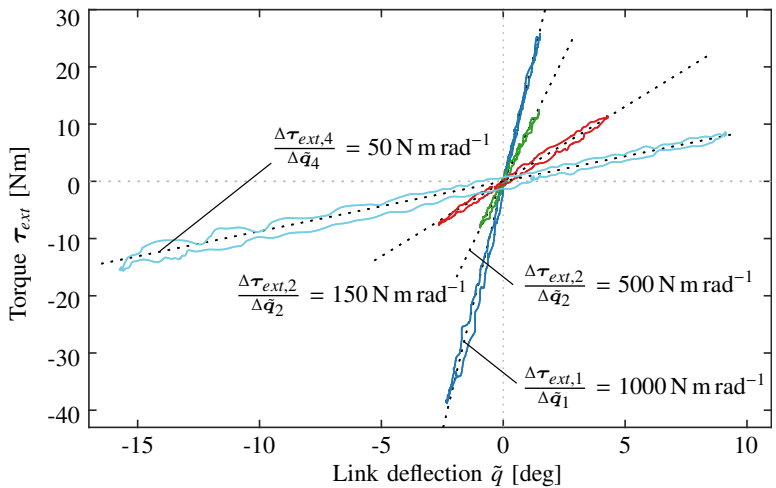

Fig. 9. Applied external torques $\boldsymbol{\tau}_{\text {ext }}$ versus link deflection $\tilde{\boldsymbol{q}}$. The black dashed lines correspond to each of the desired stiffness behaviors for the quasi-static case. 


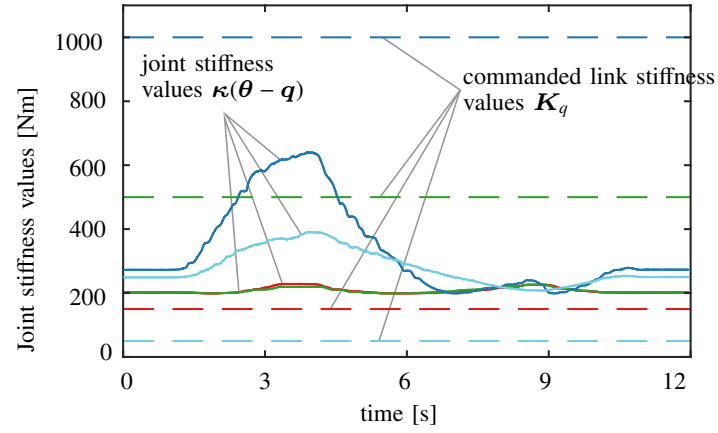

Fig. 10. Local joint stiffness values for the real physical springs.

the control error $\tilde{\boldsymbol{x}}$ converged, the internal motor movements simultaneously come to rest. We repeated this experiment with a joint-level ES $\pi$ controller. The controller gains are given in the second row of Tab. II and the results are contained in Fig. 11. This experiments shows, that even in the case of control input saturations, the controlled system stays stable.

\section{Discussion}

As the results above show, ES $\pi$ control shows impressive performance in practice (see also the attached Video):

- Excellent damping performance with basically no overshooting.

- Very precise stiffness control in joint and Cartesian space. Linear force-stiffness relations can be realized even for large displacements and not just locally.

- The link stiffness can be substantially increased over the natural stiffness of the system.

- The system stays stable even in the case of control input saturation. (see Fig. 12).

- The systems stays stable even with all damping gains set to zero (see Experiment I).

\section{Extension to the Joint-Level Tracking Case}

ES $\pi$ control based link-side motion tracking can be achieved by substituting $\boldsymbol{\tau}_{x}$ and $\boldsymbol{n}$ in (11) and (13), respectively, by

$$
\tilde{\boldsymbol{\tau}}_{q}(\tilde{\boldsymbol{q}}, \dot{\tilde{\boldsymbol{q}}})=-\boldsymbol{D}_{q} \dot{\tilde{\boldsymbol{q}}}-\left(\frac{\partial U_{c}(\tilde{\boldsymbol{q}})}{\partial \tilde{\boldsymbol{q}}}\right)^{\mathrm{T}}
$$

$$
\tilde{\boldsymbol{n}}(t, \boldsymbol{q}, \dot{\boldsymbol{q}})=\boldsymbol{g}(\boldsymbol{q})+\boldsymbol{M}(\boldsymbol{q}) \ddot{\boldsymbol{q}}_{d}(t)+\boldsymbol{C}(\boldsymbol{q}, \dot{\boldsymbol{q}}) \dot{\boldsymbol{q}}_{d}(t)+\tilde{\boldsymbol{\tau}}_{q}^{*}(t, \tilde{\boldsymbol{q}}, \dot{\tilde{\boldsymbol{q}}}),
$$

with $\tilde{\boldsymbol{q}}:=\boldsymbol{q}-\boldsymbol{q}_{d}(t)$, where $\boldsymbol{q}_{d} \in C^{n}$ is the desired trajectory, and modifying the coordinate transformation (13) to

$$
\psi(\theta-q)=\psi(\eta-\tilde{q})+\tilde{n},
$$

and otherwise proceeding analogously to Sec. III-B.

\section{Conclusion}

This paper presents an impedance control concept for robots with highly elastic joints with possibly nonlinear stiffness characteristics. It preserves the inherent physical structure of the system by preserving the inertial properties and the elastic structure of the plant. This is achieved by introducing new motor coordinates that reflect the desired link-side impedance behavior. The physically motivated nature of the design approach allows us to visualize the representing closed-loop dynamics as multi-spring-damper
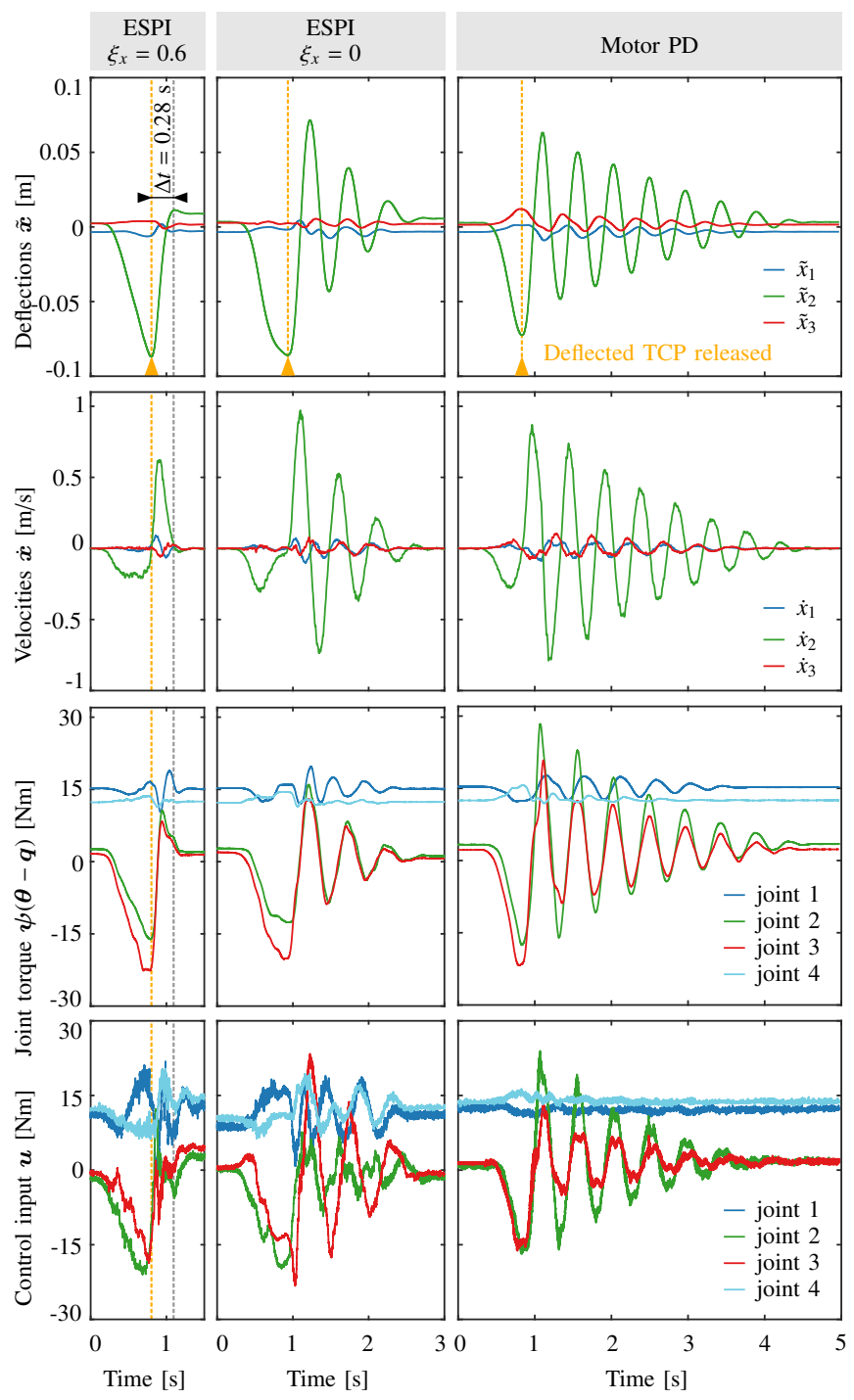

Fig. 11. The disturbance rejection performance of the ES $\pi$ controller for two different modal damping factors is shown. As reference, and in order to show the 'natural' oscillatory behavior of the system, the performance of the well known motor PD controller by Tomei is shown [25].

system. This allows for a physically intuitive interpretation of the closed-loop behavior, which in turn, turns out to be highly valuable for the tuning stage. All gains can be interpreted as spring and dampers, respectively. It also allows for a comprehensive stability and passivity analysis based on a physically motivated Lyapunov and storage function. Neither upper nor lower bounds on the gains are imposed. A theoretical limitation of the approach seemed to be the dependence on the time derivatives of the link positions up to the third order. However, in practice it turned out to be no limitation, since the time derivatives could be computer based on the model of the plant. To the best of knowledge, this is the first Cartesian impedance controller that has been experimentally validated on multi-joint robot with highly elastic variable stiffness joints featuring highly nonlinear stiffness characteristics.

We presented a Cartesian impedance control approach which provides highly elastic robots with the capability to stably interact with the environment. In theory, however, our concept allows to impose arbitrary torques on the links (un- 
Deflection $\tilde{\boldsymbol{q}}[\mathrm{deg}]$

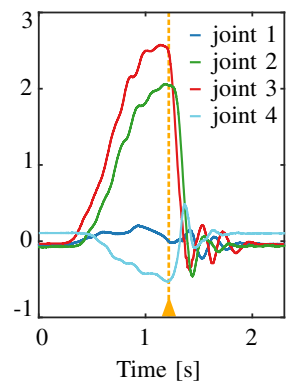

External Torques $\boldsymbol{\tau}_{\text {ext }}[\mathrm{Nm}] \quad$ Control input $\boldsymbol{u}[\mathrm{Nm}]$

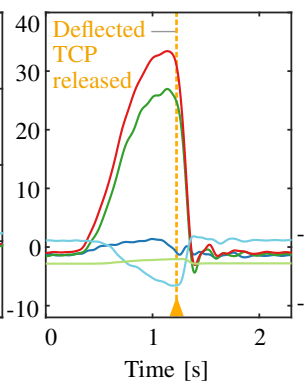

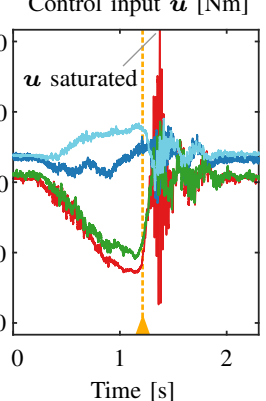

Fig. 12. The system stays stable even in case of control input saturation.

der the assumption that their first and second time derivatives are known). In future, we hope to exploit this torque interface in order to adopt classic control methods that have been originally developed for rigid robots. Thereby, we hope to further increase the field of application for highly elastic robots by providing them with abilities rigid robots robots already possess.

\section{Appendix}

Assumption 3. The positive definite spring potential function $U_{s}: \mathbb{R}^{n} \rightarrow \mathbb{R}$ is three times continuously differentiable. The elastic torque function $\psi: \mathbb{R}^{n} \rightarrow \mathbb{R}^{n}$ is defined as follows

$$
\psi(\phi):=\left(\frac{\partial U_{s}(\phi)}{\partial \phi}\right)^{T} \in \mathbb{R}^{n} .
$$

The local stiffness, i.e., the Hessian of the spring potential $U_{s}$ is non-singular for all $\phi_{0} \in \mathbb{R}^{n}$ and denoted as

$$
\kappa(\phi):=\frac{\partial \psi(\phi)}{\partial \phi} \in \mathbb{R}^{n \times n},
$$

Basically, this includes all practically relevant spring types (buckling springs excluded).

\section{A. Assumptions for the Cartesian Impedance Control Case}

We assume the existence of a $C^{3}$ forward kinematics mapping $G: \overline{\mathcal{D}}_{q} \rightarrow \overline{\mathcal{D}}_{x}$ from the link configuration variables $\boldsymbol{q} \in \overline{\mathcal{D}}_{q} \subseteq \mathbb{R}^{n}$ to the task coordinates $\boldsymbol{x} \in \overline{\mathcal{D}}_{x} \subseteq \mathbb{R}^{m}$, which, e.g., describe the position and orientation of the robot endeffector, i.e.,

$$
x=G(q) .
$$

Throughout this paper, when referring to Cartesian impedance control, we consider the non-redundant case $n=$ $m$, and assume that the Jacobian matrix of the mapping (27)

$$
\boldsymbol{J}(\boldsymbol{q})=\frac{\partial \boldsymbol{G}(\boldsymbol{q})}{\partial \boldsymbol{q}}
$$

is non-singular for all $\boldsymbol{q} \in \overline{\mathcal{D}}_{q}$, i.e., $\sup _{\boldsymbol{q} \in D_{q}}\left\|\boldsymbol{J}(\boldsymbol{q})^{-1}\right\|<\infty$, such that $G$ is a diffeomorphism [26, p. 174]. Therefore, $\boldsymbol{q}$ and $\boldsymbol{x}$ are equivalent representations of the link-side configuration.

\section{REFERENCES}

[1] M. W. Spong, "Modeling and control of elastic joint robots," Transactions of the ASME: Journal of Dynamic Systems, Measurement, and Control, vol. 109, pp. 310-319, 1987.

[2] A. Loria and R. Ortega, "On tracking control of rigid and flexible joints robots," Appl. Math. Comput. Sci, vol. 5, no. 2, pp. 101-113, 1995.
[3] C. Ott, A. Albu-Schäffer, A. Kugi, and G. Hirzinger, "Decoupling based cartesian impedance control of flexible joint robots," in Proc. IEEE Int. Conf. on Robotics and Automation, 2003.

[4] S. Ozgoli and H. Taghirad, "A survey on the control of flexible join robots," Asian Journal of Control, vol. 8, no. 4, pp. 332-344, 2006.

[5] A. De Luca and P. Lucibello, "A general algorithm for dynamic feedback linearization of robots with elastic joints," in IEEE Int. Conf on Robotics and Automation, vol. 1, 1998, pp. 504-510.

[6] A. Albu-Schäffer, O. Eiberger, M. Fuchs, M. Grebenstein, S. Haddadin, C. Ott, A. Stemmer, T. Wimböck, S. Wolf, C. Borst, an G. Hirzinger, "Anthropomorphic soft robotics - from torque control to variable intrinsic compliance," in Robotics Research, ser. Springer Tracts in Advanced Robotics. Springer, 2011, vol. 70, pp. 185-207.

[7] S. Wolf, O. Eiberger, and G. Hirzinger, "The dlr fsj: Energy based design of a variable stiffness joint," in Robotics and Automation (ICRA), 2011 IEEE International Conference on, May 2011, pp. 5082 5089.

[8] A. Albu-Schäffer, C. Ott, and G. Hirzinger, "A unified passivitybased control framework for position, torque and impedance control of flexible joint robots," The International Journal of Robotics Research, vol. 26, no. 1, pp. 23-39, 2007.

[9] C. Ott, A. Albu-Schaffer, A. Kugi, and G. Hirzinger, "On the passivitybased impedance control of flexible joint robots," Robotics, IEEE Transactions on, vol. 24, no. 2, pp. 416-429, 2008

[10] G. Pratt and M. Williamson, "Series elastic actuators," in Proceedings 1995 IEEE/RSJ International Conference on Intelligent Robots and Systems. Human Robot Interaction and Cooperative Robots. IEEE Comput. Soc. Press, 1995.

[11] D. J. Braun, M. Howard, and S. Vijayakumar, "Exploiting variable stiffness in explosive movement tasks," in Robotics: Science and Systems, 2011.

[12] M. Keppler, D. Lakatos, C. Ott, and A. Albu-Schäffer, "A passivitybased controller for motion tracking and damping assignment for compliantly actuated robots," in Decision and Control (CDC), 2016 IEEE 55th Conference on. IEEE, 2016, pp. 1521-1528.

[13] M. Keppler, D. Lakatos, C. Ott, and A. Albu-Schäffer, "Elastic structure preserving (esp) control for compliantly actuated robots,' 2018, accepted for publication in the IEEE Transactions on Robotics.

[14] A. De Luca and F. Flacco, "Dynamic gravity cancellation in robots with flexible transmissions," in Decision and Control (CDC), 2010 49th IEEE Conference on, Dec 2010, pp. 288-295.

[15] M. Keppler, D. Lakatos, C. Ott, and A. Albu-Schäffer, "Viscoelastic structure preserving impedance (vespi) control for compliantly actuated robots," 2018, accepted for publication on 2018 European Control Conference (ECC).

[16] N. Hogan, "Impedance control: An approach to manipulation: Part i-theory," Journal of Dynamic Systems, Measurement, and Control, vol. 107 , no. 1 , p. 1,1985

[17] J.-J. Slotine and W. Li, Applied Nonlinear Control. Prentice Hall, 1991.

[18] F. Ghorbel, B. Srinivasan, and M. W. Spong, "On the uniform boundedness of the inertia matrix of serial robot manipulators," Journal of Robotic Systems, vol. 15, no. 1, 1998.

[19] M. W. Spong, "Adaptive control of flexible joint manipulators," Systems $\mathcal{E}$ Control Letters, vol. 13, no. 1, pp. 15-21, 1989.

[20] D. Koditschek, "Natural motion for robot arms," in The 23rd IEEE Conference on Decision and Control. IEEE, dec 1984.

[21] O. Khatib, "A Unified Approach for Motion and Force Control of Robot Manipulators: The Operational Space Formulation," IEEE Journal of Robotics and Automation, vol. RA-3, no. 1, pp. 43-53, February 1987

[22] A. Albu-Schäffer, C. Ott, and F. Petit, "Constructive energy shaping control for a class of euler-lagrange systems," in 10th Int. IFAC Symposium on Robot Control, 2012.

[23] A. Luca, A. Albu-Schaffer, S. Haddadin, and G. Hirzinger, "Collision detection and safe reaction with the DLR-III lightweight manipulator arm," in 2006 IEEE/RSJ International Conference on Intelligent Robots and Systems. IEEE, oct 2006.

[24] M. Keppler, D. Lakatos, C. Ott, and A. Albu-Schäffer, "A passivitybased approach for trajectory tracking and link-side damping of compliantly actuated robots," in 2016 IEEE International Conference on Robotics and Automation (ICRA). IEEE, 2016, pp. 1079-1086.

[25] P. Tomei, "A simple pd controller for robots with elastic joints," Automatic Control, IEEE Transactions on, vol. 36, no. 10, pp. 12081213, Oct 1991.

[26] E. Zeidler, Nonlinear Functional Analysis and its AApplication: FixedPoint Theorems. Springer Science, 1986, vol. 1. 